\title{
SNP-SNP Interaction between TLR4 and MyD88 in Susceptibility to Coronary Artery Disease in the Chinese Han Population
}

\author{
Dandan Sun $1,2,3$, Liping Sun ${ }^{1,2}$, Qian $X u^{1,2}$, Yuehua Gong ${ }^{1,2}$, Honghu Wang ${ }^{3}$, Jun Yang ${ }^{3}$ and \\ Yuan Yuan ${ }^{1,2, *}$ \\ 1 Department of Tumor Etiology and Screening, Cancer Institute and General Surgery, The First Affiliated \\ Hospital of China Medical University, Shenyang 110001, China; dan_101912@163.com (D.S.); \\ lipingsun@hotmail.com (L.S.); xuqian820101@163.com (Q.X.); gongyueh75@163.com (Y.G.) \\ 2 Key Laboratory of Cancer Etiology and Prevention, Liaoning Provincial Education Department, China \\ Medical University, Shenyang 110001, China \\ 3 Department of Cardiovascular Ultrasound, The First Affiliated Hospital of China Medical University, \\ Shenyang 110001, China; wanghonghuzgykd@126.com (H.W.); junyang63@sina.com (J.Y.) \\ * Correspondence: yyuan@mail.cmu.edu.cn; Tel.: +86-24-8328-2153; Fax: +86-24-8328-2292
}

Academic Editor: Paul B. Tchounwou

Received: 20 November 2015; Accepted: 24 February 2016; Published: 4 March 2016

\begin{abstract}
The toll-like receptor 4 (TLR4)-myeloid differentiation factor 88 (MyD88)-dependent signaling pathway plays a role in the initiation and progression of coronary artery disease (CAD). We investigated SNP-SNP interactions between the TLR4 and MyD88 genes in CAD susceptibility and assessed whether the effects of such interactions were modified by confounding risk factors (hyperglycemia, hyperlipidemia and Helicobacter pylori (H. pylori) infection). Participants with CAD $(n=424)$ and controls $(n=424)$ without CAD were enrolled. Polymerase chain restriction-restriction fragment length polymorphism was performed on genomic DNA to detect polymorphisms in TLR4 (rs10116253, rs10983755, and rs11536889) and MyD88 (rs7744). H. pylori infections were evaluated by enzyme-linked immunosorbent assays, and the cardiovascular risk factors for each subject were evaluated clinically. The significant interaction between TLR4 rs11536889 and MyD88 rs7744 was associated with an increased CAD risk ( $p$ value for interaction $=0.024)$. In conditions of hyperglycemia, the interaction effect was strengthened between TLR4 rs11536889 and MyD88 rs7744 ( $p$ value for interaction $=0.004$ ). In hyperlipidemic participants, the interaction strength was also enhanced for TLR4 rs11536889 and MyD88 rs7744 ( $p$ value for interaction $=0.006$ ). Thus, the novel interaction between TLR4 rs11536889 and MyD88 rs7744 was related with an increased risk of CAD, that could be strengthened by the presence of hyperglycemia or hyperlipidemia.
\end{abstract}

Keywords: toll-like receptor 4; myeloid differentiation factor 88; polymorphism; Interaction; coronary artery disease

\section{Introduction}

Coronary artery disease (CAD) is the most common cause of morbidity and mortality in China [1]. It is a complex disease determined by genetic predisposition and environmental factor accumulation, which play major roles in a number of associated vessel wall abnormalities [2]. A person's genetic make-up as well as other well-known major risk factors are important for the initiation and progression of CAD. Indeed, a substantial body of literature has investigated the association of CAD with gene polymorphisms [3-5].

Toll-like receptor 4 (TLR4) and myeloid differentiation factor 88 (MyD88), which act as the gate of the innate immune system and the trigger of the adaptive immune system, have been extensively 
studied for their roles in the pathogenesis and progression of CAD [6,7]. Compared to the wild-type mice, the mice deficiency of the TLR4 gene or MyD88 gene exhibited significantly smaller infarctions, as well as lower levels of some atherogenic cytokines (e.g., IL-1 $\beta$, IL-6, and TNF $\alpha$ ) [8]. Some studies have found that a coding polymorphism in the TLR4 gene was associated with CAD or acute myocardial infarction in a Caucasian population, but not in a Chinese population [9]. Regarding the MyD88 gene, a single nucleotide polymorphism (SNP) in its $3^{\prime}$-untranslated region ( $3^{\prime}$-UTR) has been reported to be associated with Buerger disease but not with Takayasu arteritis in the Japanese population [10]. Up to date, 153 suggestive DNA variants associated with CAD have been discovered by genome-wide association study (GWAS) worldwide. However, each variant usually confers a minimal to modest increase in relative risk, averaging only $18 \%$ (corresponding to an odds ratio of 1.18) [11]. Accordingly, the results of genetic polymorphism studies that have sought to identify relationships for TLR4 and MyD88 genes with CAD remain controversial and inconclusive. In most studies, the association between the risks of $\mathrm{CAD}$ and genetic polymorphisms was often limited to one loci or haplotypes over several neighboring loci in one gene of interest, which seems insufficient as the genetic baseis for CAD is complex and varied [12]. Thus, an increasing number of studies have assessed epistatic gene-gene interaction effects on CAD susceptibility [13,14]. TLR4 is an important membrane receptor, which not only can recognize most of exogenous ligands, like lipopolysaccharide (LPS) of Helicobacter pylori (H. pylori) [15], but also can bind to some endogenous ligands, such as fetuin-A (FetA) related to hyperglycemia and minimally modified low density lipoprotein (mmLDL) involved in hyperlipidemia $[16,17]$. Thus, we made further efforts on evaluation of the modified function of the related environmental factors to the SNP-SNP interaction effect of TLR4 and MyD88 genes that are not.

Consequently, in this study, we investigated potential SNP-SNP interactions of TLR4 and MyD88 genes for their possible roles in susceptibility to CAD. We assessed whether the effects of such interactions were modified by environmental factors, such as hyperglycemia, hyperlipidemia and H. pylori infection, in order to determine the architecture of CAD predisposition and thereby improve personalized preventative for individuals at risk of this disease.

\section{Materials and Methods}

\subsection{Study Population}

This was a single center, case-control study. We collected data from 848 consecutive participants who had undergone coronary angiography at the First Affiliated Hospital of China Medical University between 2012 and 2015. This study was approved by the Ethics Committee (Ethic Approval Number: [2011]18 and 2015-68-2). Patients who had at least one vessel with stenosis of no less than $50 \%$ diameter were defined as CAD cases $(n=424)$. Those who had no demonstrable lesions on angiography served as controls $(n=424)$. The exclusion criteria were as follows: participants with cardiomyopathy, auto-immunological disease, severe kidney or liver disease, or malignant disease.

All participants had their demographic characteristics (e.g., age, sex) recorded and were examined to determine the presence of cardiovascular risk factors. The confounding risk factors were as follows: (a) smoking: individuals who had smoked at least one cigarette per day for more than one year were classified as smokers; (b) alcohol consumption: individuals who had consumed at least one alcoholic drink a day for a minimum period of six months were defined as consumers of alcohol; (c) hypertension: individuals with systolic blood pressure $\geqslant 140 \mathrm{mmHg}$ or diastolic blood pressure $\geqslant 90 \mathrm{mmHg}$, or both, were considered hypertensive; (d) hyperglycemia: individuals with fasting plasma glucose $\geqslant 7.0 \mathrm{mmol} / \mathrm{L}$ or 2 -h plasma glucose $\geqslant 11.1 \mathrm{mmol} / \mathrm{L}$, or both, were considered hyperglycemic; (e) hyperlipidemia: participants with plasma cholesterol concentration $\geqslant 5.17 \mathrm{mmol} / \mathrm{L}$, or plasma triglyceride concentration $\geqslant 1.70 \mathrm{mmol} / \mathrm{L}$ or plasma low-density lipoprotein cholesterol concentration $\geqslant 2.58 \mathrm{mmol} / \mathrm{L}$, were considered hyperlipidemic. Details of the study group characteristics were summarized in Table 1. 
Table 1. Baseline characteristics of the study participants.

\begin{tabular}{|c|c|c|c|}
\hline Variability & Cases & Controls & $p$ Value \\
\hline \multicolumn{4}{|l|}{ Total } \\
\hline Age (year) & $59.47 \pm 10.81$ & $59.27 \pm 10.89$ & 0.790 \\
\hline \multicolumn{4}{|l|}{ Sex } \\
\hline Male (\%) & $269(63.4)$ & $258(60.8)$ & 0.436 \\
\hline Female (\%) & 155 (36.6) & $166(39.2)$ & \\
\hline \multicolumn{4}{|l|}{ H. pylori } \\
\hline Positive (\%) & $183(43.2)$ & 185 (43.7) & 0.866 \\
\hline Negative (\%) & $241(56.8)$ & $239(56.3)$ & \\
\hline \multicolumn{4}{|l|}{ Smoking } \\
\hline Yes (\%) & $166(39.2)$ & $124(29.2)$ & 0.090 \\
\hline No (\%) & $257(60.6)$ & $247(58.3)$ & \\
\hline Missing (\%) & $1(0.2)$ & $53(12.5)$ & \\
\hline \multicolumn{4}{|l|}{ Alcohol } \\
\hline \multicolumn{4}{|l|}{ consumption } \\
\hline Yes $(\%)$ & $63(14.9)$ & $67(15.8)$ & 0.230 \\
\hline No (\%) & $361(84.9)$ & 304 (71.7) & \\
\hline Missing (\%) & $1(0.2)$ & $53(12.5)$ & \\
\hline \multicolumn{4}{|l|}{ Hypertension } \\
\hline Yes (\%) & $291(68.6)$ & $244(57.5)$ & 0.001 \\
\hline No $(\%)$ & $133(31.4)$ & $180(42.5)$ & \\
\hline \multicolumn{4}{|l|}{ Hyperglycemia } \\
\hline Yes (\%) & 165 (38.9) & $101(23.8)$ & 0.000 \\
\hline No $(\%)$ & $259(61.1)$ & $323(76.2)$ & \\
\hline \multicolumn{4}{|l|}{ Hyperlipidemia } \\
\hline Yes (\%) & $227(53.5)$ & $186(43.9)$ & 0.005 \\
\hline No (\%) & 197 (46.5) & $238(56.1)$ & \\
\hline
\end{tabular}

Note: H. pylori: Helicobacter pylori.

\subsection{SNP Selection and Genotyping}

A two-step approach was performed to identify tag-SNPs in TLR4 and MyD88 genes as described previously [18]. First, tag-SNPs were selected in the combinations provided by the HapMap database (Release 27, Phase I + II + III) and Haploview software $[19,20]$. Next, the functional effects of the selected tag-SNPs were predicted by FuncPred software [21]. Accordingly, two tag-SNPs (rs10116253 and rs10983755) in the promoter region of TLR4, one tag-SNP (rs11536889) in the $3^{\prime}$-UTR of TLR4 and one tag-SNP (rs7744) in the $3^{\prime}$-UTR of MyD88 were screened.

Genomic DNA of each subject was extracted from a blood clot using standard phenol-chloroform methodology. The polymorphisms were detected using the polymerase chain restriction-restriction fragment length polymorphism (PCR-RFLP) procedure. Table S1 shows the details of the PCR-RFLP conditions of the four tag-SNPs.

\subsection{H. pylori Serology}

The concentration of serum IgG, specific for $H$. pylori was tested using an enzyme-linked immunosorbent assay (H. pylori IgG ELISA kit; BIOHIT, Helsinki, Finland). The cut-off value is 34 EIU, which is given by the standard protocol (BIOHIT, Helsinki, Finland). If the titer value was above 34 EIU, the individual was defined as H. pylori infection [22]. 


\subsection{Statistical Analyses}

All statistical analyses were performed using the SPSS 16.0 statistical software package (SPSS, Chicago, IL, USA). Discrete variables, represented as frequencies and percentages, were evaluated by Pearson's $\chi^{2}$ tests. Continuous variables, presented as mean $\pm S D$, were compared using ANOVA tests. SNP-SNP interaction effects were assessed using the likelihood-ratio tests, by comparing the fit of the logistic model that included the main effects of the environment risk factors and genotypes with a fully parameterized model [23]. Odds ratios (OR) with $95 \%$ confidence intervals (CI) were calculated as measures of associations adjusted by the confounding risk factors (age, sex, hypertension, hyperglycemia, hyperlipidemia and H. pylori infection) unless the risk factor had been used as a stratified factor. A two-side $p$ value of $<0.05$ was considered statistically significant.

\section{Results}

\subsection{Main Effect Analyses of Individual Polymorphisms in the TLR4 and MyD88}

The genotype distributions of the four SNPs studied in the control participants followed Hardy-Weinberg equilibrium (HWE) $(p>0.05)$ (Table S2). In our unpublished data, we found that of the polymorphisms in TLR4 and MyD88, the TLR4 rs10116253 polymorphism was associated with a slightly decreased risk of CAD, whereas there was no overall genetic effect for TLR4 rs10983755, TLR4 rs11536889 or MyD88 rs7744 relating to CAD risk.

\subsection{Two-Way Interactions between TLR4 and MyD88 Polymorphisms}

In the two-way interaction analyses, the most significant interaction was between TLR4 rs11536889 and MyD88 rs7744. This interaction was associated with an increased risk of CAD ( $p$ value for interaction $=0.024$, OR $(95 \% \mathrm{CI})=1.928(1.089-3.413))$. In contrast, in the two-way analyses between TLR4 rs10116253 or TLR4 rs10983755 and MyD88 rs7744, no statistically significant interactions were observed ( $p$ value for interaction $>0.05$ ) (Table 2).

\subsection{The Effect of Confounding Risk Factors on the Interaction between Polymorphisms in TLR4 and MyD88}

In stratified analyses, we tested the effect of environmental risk factors $(H$. pylori infection, hyperglycemia and hyperlipidemia) on the interaction strength (Table 3). Under conditions of hyperglycemia, the OR (95\% CI) was 4.905 (1.640-14.673) between TLR4 rs11536889 and MyD88 rs7744 $(p$ value for interaction $=0.004)$. In contrast, the OR $(95 \% \mathrm{CI})$ was $1.336(0.664-2.686)$ for the participants with normal plasma glucose levels ( $p$ value for interaction $=0.417$ ). Moreover, when the participants had hyperlipidemia, the OR (95\% CI) was 3.269 (1.398-7.644) between TLR4 rs11536889 and MyD88 rs7744 ( $p$ value for interaction $=0.006$ ). However, no interaction effect was noted in the participants who lacked hyperlipidemia $(\mathrm{OR}(95 \% \mathrm{CI})=1.156(0.513-2.604)$, $p$ value for interaction $=0.726$ ). Furthermore, $H$. pylori infection did not influence the interaction effect between TLR4 rs11536889 and MyD88 rs7744 for CAD risk ( $p$ value for interaction $>0.05$ ). As to the analyses between TLR4 rs10116253 or TLR4 rs10983755 and MyD88 rs7744, no modification by any of the environmental risk factors was identified ( $p$ value for interaction $>0.05$ ) (Tables 4 and 5). 
Table 2. Two-way interactions between TLR4 and MyD88 polymorphisms in the risk of CAD.

\begin{tabular}{|c|c|c|c|c|c|c|}
\hline \multirow{2}{*}{ TLR4 } & \multirow{2}{*}{ Genotypes } & \multirow{2}{*}{ Number of Participants } & \multicolumn{4}{|c|}{ MyD88 rs7744 } \\
\hline & & & AA & $\mathrm{AG}+\mathrm{GG}$ & $\mathrm{AA}+\mathrm{AG}$ & GG \\
\hline \multirow{10}{*}{ rs10116253 } & $\mathrm{TC}+\mathrm{CC}$ & No. of controls/cases & $123 / 101$ & $179 / 169$ & $258 / 242$ & $44 / 28$ \\
\hline & & OR $(95 \% \mathrm{CI})$ & 1.0 (ref.) & $1.052(0.599-1.848)$ & 1.0 (ref.) & $0.715(0.427-1.197)$ \\
\hline & TT & No. of controls/cases & $51 / 56$ & $71 / 98$ & $101 / 128$ & $21 / 26$ \\
\hline & & OR $(95 \%$ CI $)$ & $1.158(0.608-2.207)$ & $1.561(0.844-2.887)$ & $1.313(0.951-1.812)$ & $1.406(0.762-2.592)$ \\
\hline & & & \multicolumn{2}{|c|}{$p=0.654$, OR $(95 \% \mathrm{CI})=1.148(0.627-2.104)$} & \multicolumn{2}{|c|}{$p=0.322, \mathrm{OR}(95 \% \mathrm{CI})=1.517(0.665-3.463)$} \\
\hline & $\mathrm{CC}$ & No. of controls/cases & $31 / 29$ & $51 / 42$ & $67 / 67$ & $15 / 4$ \\
\hline & & OR $(95 \% \mathrm{CI})$ & 1.0 (ref.) & $0.859(0.433-1.702)$ & 1.0 (ref.) & $0.338(0.103-1.106)$ \\
\hline & $\mathrm{TC}+\mathrm{TT}$ & No. of controls/cases & $143 / 128$ & $143 / 225$ & $288 / 303$ & $54 / 50$ \\
\hline & & OR $(95 \% \mathrm{CI})$ & $0.982(0.558-1.728)$ & $1.290(0.739-2.252)$ & $1.078(0.735-1.581)$ & $1.121(0.651-1.931)$ \\
\hline & & & \multicolumn{2}{|c|}{$p=0.347$, OR $(95 \%$ CI $)=1.423(0.682-2.966)$} & \multicolumn{2}{|c|}{$p=0.065$, OR $(95 \%$ CI $)=3.231(0.929-11.236)$} \\
\hline \multirow{10}{*}{ rs10983755 } & $\mathrm{GA}+\mathrm{AA}$ & No. of controls/cases & $94 / 76$ & $141 / 130$ & $203 / 184$ & $32 / 22$ \\
\hline & & OR $(95 \% \mathrm{CI})$ & 1.0 (ref.) & $1.110(0.749-1.644)$ & 1.0 (ref.) & $0.774(0.429-1.395)$ \\
\hline & GG & No. of controls/cases & $80 / 81$ & $109 / 137$ & $156 / 186$ & $33 / 32$ \\
\hline & & OR $(95 \% \mathrm{CI})$ & $1.214(0.781-1.887)$ & $1.595(1.061-2.396)$ & $1.292(0.959-1.741)$ & $1.139(0.667-1.945)$ \\
\hline & & & \multicolumn{2}{|c|}{$p=0.612$, OR $(95 \% \mathrm{CI})=1.158(0.657-2.042)$} & \multicolumn{2}{|c|}{$p=0.728$, OR $(95 \% \mathrm{CI})=1.153(0.517-2.570)$} \\
\hline & AA & No. of controls/cases & $14 / 14$ & $24 / 22$ & $30 / 35$ & $8 / 1$ \\
\hline & & OR $(95 \% \mathrm{CI})$ & 1.0 (ref.) & $0.890(0.312-2.541)$ & 1.0 (ref.) & $0.125(0.013-1.217)$ \\
\hline & $\mathrm{GA}+\mathrm{GG}$ & No. of controls/cases & $160 / 143$ & $226 / 245$ & $325 / 335$ & $61 / 53$ \\
\hline & & OR $(95 \% \mathrm{CI})$ & $0.997(0.453-2.195)$ & $1.287(0.587-2.822)$ & $0.947(0.562-1.596)$ & $0.908(0.471-1.752)$ \\
\hline & & & \multicolumn{2}{|c|}{$p=0.777$, OR $(95 \%$ CI $)=1.156(0.424-3.157)$} & \multicolumn{2}{|c|}{$p=0.074, \mathrm{OR}(95 \% \mathrm{CI})=7.346(0.823-65.536)$} \\
\hline \multirow{10}{*}{ rs11536889 } & GG & No. of controls/cases & $100 / 99$ & $170 / 156$ & $224 / 221$ & $46 / 34$ \\
\hline & & OR $(95 \% \mathrm{CI})$ & 1.0 (ref.) & $0.930(0.653-1.323)$ & 1.0 (ref.) & $0.793(0.485-1.297)$ \\
\hline & $\mathrm{GC}+\mathrm{CC}$ & No. of controls/cases & $74 / 58$ & $80 / 111$ & $135 / 149$ & $19 / 20$ \\
\hline & & OR $(95 \%$ CI $)$ & $0.782(0.501-1.220)$ & $1.395(0.934-2.085)$ & $1.123(0.828-1.522)$ & $1.258(0.640-2.471)$ \\
\hline & & & \multicolumn{2}{|c|}{$p=0.024, \mathrm{OR}(95 \% \mathrm{CI})=1.928(1.089-3.413)$} & \multicolumn{2}{|c|}{$p=0.436$, OR $(95 \%$ CI $)=1.399(0.601-3.258)$} \\
\hline & GG + GC & No. of controls/cases & $165 / 152$ & $238 / 247$ & $345 / 352$ & $58 / 47$ \\
\hline & \multirow{4}{*}{$\mathrm{CC}$} & OR $(95 \% \mathrm{CI})$ & 1.0 (ref.) & $1.143(0.854-1.529)$ & 1.0 (ref.) & $0.851(0.558-1.298)$ \\
\hline & & No. of controls/cases & $9 / 5$ & $12 / 20$ & $14 / 18$ & $7 / 7$ \\
\hline & & OR $(95 \% \mathrm{CI})$ & $0.589(0.192-1.810)$ & $1.884(0.876-4.052)$ & $1.237(0.598-2.561)$ & $1.113(0.379-3.267)$ \\
\hline & & & $p=0.119$, OR & $943(0.756-11.454)$ & $p=0.909$, OR & $.082(0.280-4.181)$ \\
\hline
\end{tabular}

Notes: All tests were adjusted by age, sex, hypertension, hyperglycemia, hyperlipidemia and $H$. pylori infection. Statistically significant interaction was highlighted in bold $(p$ value for interaction <0.05). CAD: coronary artery disease; TLR4: toll-like receptor 4; MyD88: myeloid differentiation factor 88; OR: odds ratio; CI: confidence interval; ref.: reference. 
Table 3. The effect of confounding risk factors on the interaction between TLR4 rs11536889 and MyD88 rs7744 in the risk of CAD.

\begin{tabular}{|c|c|c|c|c|c|c|c|c|c|}
\hline \multirow{2}{*}{$\begin{array}{c}\text { TLR4 } \\
\text { rs11536889 }\end{array}$} & \multirow{2}{*}{$\begin{array}{c}M y D 88 \\
\text { rs7744 }\end{array}$} & \multirow{2}{*}{ Controls $(n)$} & \multirow{2}{*}{ Cases $(n)$} & \multicolumn{2}{|c|}{ Cases vs. Controls } & \multirow{2}{*}{ Controls $(n)$} & \multirow{2}{*}{ Cases $(n)$} & \multicolumn{2}{|c|}{ Cases vs. Controls } \\
\hline & & & & OR $(95 \% \mathrm{CI})$ & $p$ & & & OR $(95 \% \mathrm{CI})$ & $p$ \\
\hline & & \multicolumn{4}{|c|}{ H. pylori $(-)^{\mathrm{a}}$} & \multicolumn{4}{|c|}{ H. pylori $(+)^{\mathrm{a}}$} \\
\hline GG & AA & 55 & 53 & 1 (ref.) & & 45 & 46 & 1 (ref.) & \\
\hline GG & $\mathrm{AG}+\mathrm{GG}$ & 95 & 86 & $0.954(0.582-1.564)$ & 0.851 & 75 & 70 & $0.963(0.558-1.662)$ & 0.892 \\
\hline $\mathrm{GC}+\mathrm{CC}$ & AA & 45 & 35 & $0.829(0.456-1.510)$ & 0.541 & 29 & 23 & $0.784(0.386-1.592)$ & 0.501 \\
\hline \multirow[t]{2}{*}{$\mathrm{GC}+\mathrm{CC}$} & $\mathrm{AG}+\mathrm{GG}$ & 44 & 67 & $1.683(0.959-2.956)$ & 0.070 & 36 & 44 & $1.180(0.609-2.287)$ & 0.624 \\
\hline & & \multicolumn{4}{|c|}{$p=0.065, \mathrm{OR}(95 \% \mathrm{CI})=2.078(0.956-4.517)$} & & & \multicolumn{2}{|c|}{$p=0.354, \mathrm{OR}(95 \% \mathrm{CI})=1.531(0.621-3.773)$} \\
\hline & & \multirow{2}{*}{\multicolumn{4}{|c|}{ Hyperglycemia $(-)^{b}$}} & \multicolumn{4}{|c|}{ Hyperglycemia $(+)^{b}$} \\
\hline GG & AA & & & & & 24 & 39 & 1 (ref.) & \\
\hline GG & $\mathrm{AG}+\mathrm{GG}$ & 128 & 97 & $0.969(0.626-1.501)$ & 0.890 & 42 & 59 & $0.948(0.488-1.842)$ & 0.875 \\
\hline $\mathrm{GC}+\mathrm{CC}$ & AA & 54 & 42 & $0.967(0.566-1.650)$ & 0.902 & 20 & 16 & $0.477(0.200-1.137)$ & 0.095 \\
\hline \multirow[t]{2}{*}{$\mathrm{GC}+\mathrm{CC}$} & $\mathrm{AG}+\mathrm{GG}$ & 65 & 60 & $1.240(0.748-2.055)$ & 0.404 & 15 & 51 & $2.265(1.024-5.011)$ & 0.044 \\
\hline & & \multicolumn{4}{|c|}{$p=0.417, \mathrm{OR}(95 \% \mathrm{CI})=1.336(0.664-2.686)$} & \multicolumn{4}{|c|}{$p=0.004, \mathrm{OR}(95 \% \mathrm{CI})=4.905(1.640-14.673$} \\
\hline & & \multicolumn{4}{|c|}{ Hyperlipidemia $(-)^{c}$} & \multicolumn{4}{|c|}{ Hyperlipidemia $(+)^{\mathrm{c}}$} \\
\hline GG & AA & 60 & 46 & 1 (ref.) & & 40 & 53 & 1 (ref.) & \\
\hline GG & $\mathrm{AG}+\mathrm{GG}$ & 91 & 67 & $0.989(0.594-1.647)$ & 0.968 & 79 & 89 & $0.930(0.551-1.571)$ & 0.787 \\
\hline $\mathrm{GC}+\mathrm{CC}$ & AA & 36 & 32 & $1.152(0.621-2.136)$ & 0.653 & 38 & 26 & $0.569(0.293-1.107)$ & 0.097 \\
\hline \multirow[t]{2}{*}{$\mathrm{GC}+\mathrm{CC}$} & $\mathrm{AG}+\mathrm{GG}$ & 51 & 52 & $1.390(0.782-2.471)$ & 0.261 & 29 & 59 & $1.613(0.859-3.029)$ & 0.137 \\
\hline & & \multicolumn{4}{|c|}{$p=0.726, \mathrm{OR}(95 \% \mathrm{CI})=1.156(0.513-2.604)$} & & & \multicolumn{2}{|c|}{$p=0.006$, OR $(95 \%$ CI $)=3.269(1.398-7.644)$} \\
\hline
\end{tabular}

a , these tests were adjusted by age, sex, hypertension, hyperglycemia and hyperlipidemia; ${ }^{\text {b }}$, these tests were adjusted by age, sex, hypertension, hyperlipidemia and $H$. pylori infection;

$c$, these tests were adjusted by age, sex, hypertension, hyperglycemia and H. pylori infection. Statistically significant interactions were highlighted in bold ( $p$ value for interaction $<0.05$ )

CAD: coronary artery disease; TLR4: toll-like receptor 4; MyD88: myeloid differentiation factor 88; H. pylori: Helicobacter pylori; OR: odds ratio; CI: confidence interval. 
Table 4. The effect of confounding risk factors on the interaction between TLR4 rs10116253 and MyD88 rs7744 in the risk of CAD.

\begin{tabular}{|c|c|c|c|c|c|c|c|c|c|}
\hline \multirow{2}{*}{$\begin{array}{c}\text { TLR4 } \\
\text { rs10116253 }\end{array}$} & \multirow{2}{*}{$\begin{array}{c}M y D 88 \\
\text { rs7744 }\end{array}$} & \multirow{2}{*}{ Controls $(n)$} & \multirow{2}{*}{ Cases $(n)$} & \multicolumn{2}{|c|}{ Cases vs. Controls } & \multirow{2}{*}{ Controls $(n)$} & \multirow{2}{*}{ Cases $(n)$} & \multicolumn{2}{|c|}{ Cases vs. Controls } \\
\hline & & & & OR $(95 \% \mathrm{CI})$ & $p$ & & & OR $(95 \% \mathrm{CI})$ & $p$ \\
\hline & & \multicolumn{4}{|c|}{ H. pylori $(-)^{\mathrm{a}}$} & \multicolumn{4}{|c|}{ H. pylori $(+)^{\mathrm{a}}$} \\
\hline $\mathrm{TC}+\mathrm{CC}$ & AA & 69 & 62 & 1 (ref.) & & 54 & 39 & 1 (ref.) & \\
\hline $\mathrm{TC}+\mathrm{CC}$ & $\mathrm{AG}+\mathrm{GG}$ & 103 & 92 & $0.962(0.430-2.153)$ & 0.925 & 76 & 77 & $1.076(0.507-2.285)$ & 0.848 \\
\hline TT & AA & 31 & 26 & $0.903(0.361-2.260)$ & 0.828 & 20 & 30 & $1.594(0.657-3.868)$ & 0.303 \\
\hline \multirow[t]{2}{*}{ TT } & $\mathrm{AG}+\mathrm{GG}$ & 36 & 61 & $1.825(0.772-4.312)$ & 0.170 & 35 & 37 & $1.123(0.493-2.562)$ & 0.782 \\
\hline & & \multicolumn{4}{|c|}{$p=0.082, \mathrm{OR}(95 \% \mathrm{CI})=2.032(0.914-4.518)$} & & & \multicolumn{2}{|c|}{$p=0.132, \mathrm{OR}(95 \% \mathrm{CI})=0.502(0.205-1.231$} \\
\hline & & \multicolumn{4}{|c|}{ Hyperglycemia $(-)^{b}$} & \multicolumn{4}{|c|}{ Hyperglycemia $(+)^{b}$} \\
\hline $\mathrm{TC}+\mathrm{CC}$ & AA & 95 & 67 & 1 (ref.) & & 28 & 34 & 1 (ref.) & \\
\hline $\mathrm{TC}+\mathrm{CC}$ & $\mathrm{AG}+\mathrm{GG}$ & 137 & 102 & $1.041(0.518-2.092)$ & 0.910 & 42 & 67 & $1.384(0.511-3.752)$ & 0.523 \\
\hline TT & AA & 35 & 35 & $1.243(0.556-2.781)$ & 0.597 & 16 & 21 & $0.955(0.3026-3.023)$ & 0.937 \\
\hline \multirow[t]{2}{*}{ TT } & $\mathrm{AG}+\mathrm{GG}$ & 56 & 55 & $1.349(0.633-2.875)$ & 0.439 & 15 & 43 & $2.744(0.903-8.345)$ & 0.075 \\
\hline & & \multicolumn{4}{|c|}{$p=0.863, \mathrm{OR}(95 \% \mathrm{CI})=0.938(0.450-1.953)$} & \multicolumn{4}{|c|}{$p=0.348, \mathrm{OR}(95 \% \mathrm{CI})=1.683(0.567-4.992)$} \\
\hline & & \multicolumn{4}{|c|}{ Hyperlipidemia $(-)^{\mathrm{c}}$} & \multicolumn{4}{|c|}{ Hyperlipidemia $(+)^{\mathrm{c}}$} \\
\hline $\mathrm{TC}+\mathrm{CC}$ & AA & 68 & 52 & 1 (ref.) & & 55 & 49 & 1 (ref.) & \\
\hline $\mathrm{TC}+\mathrm{CC}$ & $\mathrm{AG}+\mathrm{GG}$ & 102 & 71 & $1.026(0.451-2.333)$ & 0.951 & 77 & 98 & $1.215(0.542-2.720)$ & 0.637 \\
\hline TT & AA & 28 & 26 & $1.217(0.480-3.087)$ & 0.679 & 23 & 30 & $1.165(0.427-3.178)$ & 0.765 \\
\hline \multirow{2}{*}{ TT } & $\mathrm{AG}+\mathrm{GG}$ & 40 & 48 & & 0.170 & 31 & 50 & $1.513(0.624-3.667)$ & 0.359 \\
\hline & & \multicolumn{4}{|c|}{$p=0.354, \mathrm{OR}(95 \% \mathrm{CI})=1.497(0.637-3.517)$} & & & \multicolumn{2}{|c|}{$p=0.688, \mathrm{OR}(95 \% \mathrm{CI})=0.837(0.351-1.996$} \\
\hline
\end{tabular}

${ }^{a}$, these tests were adjusted by age, sex, hypertension, hyperglycemia and hyperlipidemia; ${ }^{\mathrm{b}}$, these tests were adjusted by age, sex, hypertension, hyperlipidemia and $H . p y l o r i$ infection;

$c$, these tests were adjusted by age, sex, hypertension, hyperglycemia and H. pylori infection; Statistically significant interactions were highlighted in bold ( $p$ value for interaction $<0.05$ ) CAD: coronary artery disease; TLR4: toll-like receptor 4; MyD88: myeloid differentiation factor 88; H. pylori: Helicobacter pylori; OR: odds ratio; CI: confidence interval. 
Table 5. The effect of confounding risk factors on the interaction between TLR4 rs10983755 and MyD88 rs7744 in the risk of CAD.

\begin{tabular}{|c|c|c|c|c|c|c|c|c|c|}
\hline \multirow{2}{*}{$\begin{array}{c}\text { TLR4 } \\
\text { rs10983755 }\end{array}$} & \multirow{2}{*}{$\begin{array}{c}M y D 88 \\
\text { rs7744 }\end{array}$} & \multirow{2}{*}{ Controls $(n)$} & \multirow{2}{*}{ Cases $(n)$} & \multicolumn{2}{|c|}{ Cases vs. Control } & \multirow{2}{*}{ Controls $(n)$} & \multirow{2}{*}{ Cases $(n)$} & \multicolumn{2}{|c|}{ Cases vs. Controls } \\
\hline & & & & OR $(95 \% \mathrm{CI})$ & $p$ & & & OR $(95 \% \mathrm{CI})$ & $p$ \\
\hline & & & & H. pylori $(-)^{\text {a }}$ & & & & H. pylori $(+)^{a}$ & \\
\hline $\mathrm{GA}+\mathrm{AA}$ & AA & 51 & 46 & 1 (ref.) & & 43 & 30 & 1 (ref.) & \\
\hline $\mathrm{GA}+\mathrm{AA}$ & $\mathrm{AG}+\mathrm{GG}$ & 81 & 71 & $0.972(0.583-1.619)$ & 0.913 & 60 & 59 & $1.409(0.782-2.539)$ & 0.253 \\
\hline GG & AA & 49 & 42 & $0.950(0.536-1.686)$ & 0.862 & 31 & 39 & $1.803(0.929-3.500)$ & 0.081 \\
\hline \multirow[t]{2}{*}{ GG } & $\mathrm{AG}+\mathrm{GG}$ & 58 & 82 & $1.567(0.931-2.640)$ & 0.091 & 51 & 55 & $1.546(0.847-2.823)$ & 0.156 \\
\hline & & \multicolumn{6}{|c|}{$p=0.160, \mathrm{OR}(95 \% \mathrm{CI})=1.697(0.812-3.547)$} & \multicolumn{2}{|c|}{$p=0.249, \mathrm{OR}(95 \% \mathrm{CI})=0.608(0.261-1.416$} \\
\hline & & \multirow{2}{*}{\multicolumn{4}{|c|}{ Hyperglycemia $(-)^{\mathrm{b}}$}} & \multicolumn{4}{|c|}{ Hyperglycemia $(+)^{b}$} \\
\hline $\mathrm{GA}+\mathrm{AA}$ & AA & & & & & 21 & 24 & 1 (ref.) & \\
\hline $\mathrm{GA}+\mathrm{AA}$ & $\mathrm{AG}+\mathrm{GG}$ & 106 & 77 & $1.044(0.655-1.664)$ & 0.857 & 35 & 53 & $1.362(0.644-2.881)$ & 0.419 \\
\hline GG & AA & 57 & 50 & $1.206(0.709-2.054)$ & 0.489 & 23 & 31 & $1.087(0.478-2.471)$ & 0.842 \\
\hline \multirow[t]{2}{*}{ GG } & $A G+G G$ & 87 & 80 & $1.397(0.865-2.256)$ & 0.172 & 22 & 57 & $2.407(1.097-5.284)$ & 0.029 \\
\hline & & \multicolumn{4}{|c|}{$p=0.922, \mathrm{OR}(95 \% \mathrm{CI})=1.035(0.523-2.046)$} & \multicolumn{4}{|c|}{$p=0.509, \mathrm{OR}(95 \% \mathrm{CI})=1.421(0.501-4.032$} \\
\hline & & \multicolumn{4}{|c|}{ Hyperlipidemia $(-)^{\mathrm{c}}$} & \multicolumn{4}{|c|}{ Hyperlipidemia $(+)^{\mathrm{c}}$} \\
\hline $\mathrm{GA}+\mathrm{AA}$ & AA & 49 & 40 & 1 (ref.) & & 45 & 36 & 1 (ref.) & \\
\hline $\mathrm{GA}+\mathrm{AA}$ & $\mathrm{AG}+\mathrm{GG}$ & 76 & 55 & $0.904(0.519-1.575)$ & 0.722 & 65 & 75 & $1.419(0.804-2.504)$ & 0.228 \\
\hline GG & AA & 47 & 38 & $0.979(0.532-1.802)$ & 0.946 & 33 & 43 & $1.509(0.780-2.920)$ & 0.222 \\
\hline \multirow[t]{2}{*}{ GG } & $\mathrm{AG}+\mathrm{GG}$ & 66 & 64 & $1.247(0.708-2.198)$ & 0.445 & 43 & 73 & $2.117(1.163-3.854)$ & 0.014 \\
\hline & & & & $p=0.441, \mathrm{OR}(95 \%$ & $6(0.617-3.025)$ & \multicolumn{4}{|c|}{$p=0.852, \mathrm{OR}(95 \% \mathrm{CI})=0.925(0.407-2.101$} \\
\hline
\end{tabular}

${ }^{a}$, these tests were adjusted by age, sex, hypertension, hyperglycemia and hyperlipidemia; ${ }^{\mathrm{b}}$, these tests were adjusted by age, sex, hypertension, hyperlipidemia and $H . p y l o r i$ infection;

$c$, these tests were adjusted by age, sex, hypertension, hyperglycemia and H. pylori infection; Statistically significant interactions were highlighted in bold ( $p$ value for interaction $<0.05$ ) CAD: coronary artery disease; TLR4: toll-like receptor 4; MyD88: myeloid differentiation factor 88; H. pylori: Helicobacter pylori; OR: odds ratio; CI: confidence interval. 


\section{Discussion}

Genetic polymorphisms in humans can be used to predict the risks of particular diseases occurring. However, many previous studies have focused their attention on identifying single gene polymorphisms responsible for disease risk, but often no effects or weak effects have been found in such studies $[24,25]$. Recently, increasing studies have investigated interactions among combinations of two or more SNPs, and the results have usually revealed a moderate or strong effect on disease risk [23,26]. To the best of our knowledge, this study is the first to assess the interaction effects of TLR4 and MyD88 polymorphisms on CAD risk in the Chinese Han population. TLR4, as the gate of inflammatory reaction, not only can recognize pathogen-associated molecular patterns (PAMPs), but also can initiate inflammation in the lipid-laden artery wall via the NF-kB pathway, which have been proved to take part in the initiation and progression of atherosclerosis and its related complications $[27,28]$. As to MyD88, the cytoplasmic receptor adaptor of TLR4, has been widely studied in atherogenesis. Besides involving in the classical TLR4-MyD88-dependent signaling pathway related to atheroscleorsis, MyD88 has been also played an important role in obesity-associated inflammatory diseases, including insulin resistance and atherosclerosis [29]. Hence, we performed interaction effect analyses on three tag-SNPs in TLR4 (rs10116253, rs10983755 and rs11536889) and one tag-SNP in MyD88 (rs7744) to evaluate the risk of CAD in the Chinese Han population. We found that an interaction effect between rs11536889 in TLR4 and rs7744 in MyD88 was associated with an increased risk of CAD. Furthermore, the interaction effect was exacerbated by the presence of hyperglycemia or hyperlipidemia.

Evidence is accumulating that TLR4 and MyD88 have a close relationship with many inflammation-related diseases, and many studies in recent years have focused on polymorphisms in TLR4 and MyD88 genes with disease risk [30,31]. Some researchers have reported that TLR4 rs11536889 polymorphism is associated with a variety of autoimmune diseases, such as Grave's disease and autoimmune pancreatitis [32]. A study by Wang et al. revealed a relationship between TLR4 rs11536889 and sepsis [33]. Furthermore, the results from Sato $e t$ al. indicated that genetic variation of rs 11536889 contributes to translational regulation of TLR4, possibly by binding to microRNAs [34]. Regarding MyD88 rs7744, Chen et al. found that the variant genotypes of rs7744 were associated with Buerger's disease in a Japanese population [10]. However, we found that when analyzed as a single locus, neither TLR4 rs11536889 nor MyD88 rs7744 had an effect on CAD risk. In contrast, the interaction effect of TLR4 rs11536889 and MyD88 rs7744 was associated with an increased risk of CAD. We consider the interaction effect of these two SNPs to be epistasis, which has been involved in susceptibility to various inflammation-related diseases, such as malignant tumors, asthma, and Parkinson's disease [35-37]. The epistatic effect of two or more genes can account for the missing heritability of many diseases, a phenomenon often underestimated or even ignored. Indeed, the genetic effects of TLR4 rs11536889 and MyD88 rs7744 polymorphisms on the risk of CAD would most likely have been missed had they not been tested jointly. Consequently, the epistatic effects of TLR4 rs11536889 and MyD88 rs7744 on the pathogenesis and progression of CAD might depend on the presence of the other SNP. It is assumed that a functional effect on TLR4 and MyD88 in the TLR4-MyD88-dependent signaling pathway might account for the interaction effect we observed. Any genetic mutation within this pathway, especially in key genes like TLR4 and MyD88, could potentially alter the action of other components of the pathway so as to influence inflammatory reactions in the pathogenesis and progression of atherosclerosis. Our study focused only on a few tag-SNPs with potential functions in the promoter and $3^{\prime}$-UTR of TLR4 and MyD88 genes, but this approach does not capture all genetic variants in these two genes. Therefore, further analyses covering more tag-SNPs should be undertaken to investigate the potential interaction effects of TLR4 and MyD88 more fully.

In the current study, heterogeneity in the hyperglycemia or hyperlipidemia status of the study participants had a significant effect on the interaction of TLR4 rs11536889 and MyD88 rs7744. Moreover, the interaction strength was enhanced under conditions of hyperglycemia or hyperlipidemia. Evidence suggests that exogenous and endogenous ligands can activate the TLR4-MyD88-dependent signaling pathway [38,39]. Miller et al. showed that the mmLDL-induced stimulation of macropinocytosis 
was TLR4 dependent and resulted in lipid accumulation in macrophages [17]. Pal et al. found that FetA played a crucial role in regulating insulin sensitivity via the TLR4-MyD88-dependent signaling pathway in mice. FetA knockdown in mice with hyperglycemia resulted in inactivation of the TLR4-MyD88-dependent signaling pathway, whereas selective administration of FetA induced inflammatory signaling and insulin resistance [16]. In addition, Yu et al. [29] showed MyD88-dependent interplay between myeloid and endothelial cells in the initiation and progression of atherosclerosis. MyD88 deficiency in endothelial cells results in a moderate reduction in diet-induced adipose macrophage infiltration, and M1 polarization, selective insulin sensitivity in adipose tissue, and amelioration of spontaneous atherosclerosis [29]. Therefore, we hypothesized that TLR4 and MyD88 were highly likely to be associated with hyperglycemia and hyperlipidemia, consistent with the effect-modification by hyperglycemia and hyperlipidemia that was observed in TLR4 rs11536889 and MyD88 rs7744 interaction.

Although the LPS of $H$. pylori has been shown to be one of the most powerful exogenous TLR4 ligands, there is no evidence of systemic invasion of $\mathrm{H}$. pylori beyond the intestinal mucosa. Researchers have looked for H. pylori DNA in atheromatous tissue specimens using PCR. Kaklikkaya et al. did not detect $H$. pylori DNA in 21 patients with aortoiliac occlusive disease [40]. In addition, Dore $e t$ al., found that only one of 32 atherosclerotic plaques obtained at endarterectomy was positive for H. pylori DNA; however, the possibility of contamination could not be excluded in this study [41]. Hishiki et al. have speculated that a relationship between $H$. pylori, decreased body mass index and decreased plasma total cholesterol caused by dyspepsia exists, and that eradication of $H$. pylori might exaggerate the metabolic syndrome [42]. In the present study, no interaction effect between TLR4 rs11536889 and MyD88 rs7744 polymorphisms in the subgroup analyses for $H$. pylori infection was identified. Taken together, the evidence above indicates that $H$. pylori is unlikely to be involved in the atherogenic process in arteries, and supports our findings that $H$. pylori does not influence the interaction effect of TLR4 rs11536889 and MyD88 rs7744 in CAD risk.

Our study has some limitations. Firstly, although our study comprised 424 CAD participants and 424 controls, this sample size may still be relatively insufficient for fully analyzing interaction effects. Secondly, additional adenosine functional tests were absent, so we could not investigate the relationship of SNP-SNP interaction effects on microvascular dysfunction in the participants [43]. Thirdly, some information was lost for a small number of study participants, such as lifestyle factors (i.e., smoking and alcohol status), precluding their use as environmental factors in our multivariate logistic regression. Lastly, this study was hospital-based, which might increase the selection bias in comparison with population-based study.

\section{Conclusions}

In summary, our study is the first to show that a novel SNP interaction between TLR4 rs11536889 and MyD88 rs7744 is associated with an increased risk of CAD. Furthermore, the interaction strength was enhanced under conditions of hyperglycemia or hyperlipidemia. Our results provide a potential genetic clue to help predict CAD risk in susceptible people. Large-scale studies and experiments to determine the mechanisms are required to confirm the findings of this study.

Supplementary Materials: The following are available online at www.mdpi.com/1660-4601/13/3/278/s1. Table S1. Primer sequences and reaction conditions. Table S2. The genotype frequencies and HWE in this study.

Acknowledgments: This work was supported partly by grants from the Natural Science Foundation of Liaoning Province (Ref. No. 2015020506).

Author Contributions: Conceived and designed the experiments: Yuan Yuan, Jun Yang. Performed the experiments: Dandan Sun, Honghu Wang. Analyzed the data: Dandan Sun, Liping Sun, Qian Xu. Collected the serum/biopsy samples: Dandan Sun, Yuehua Gong, Jun Yang. Contributed reagents/materials/analysis tools: Yuan Yuan. Wrote the paper: Dandan Sun, Yuan Yuan, Jun Yang.

Conflicts of Interest: All authors do not have a commercial or other association that might pose a conflict of interest. 


\section{Abbreviations}

$\begin{array}{ll}\text { TLR4 } & \text { toll-like receptor } 4 \\ \text { MyD88 } & \text { myeloid differentiation factor } 88 \\ \text { CAD } & \text { coronary artery disease } \\ \text { SNP } & \text { single nucleotide polymorphism } \\ \text { H. } & \text { Helicobacter pylori } \\ \text { By flbFrR } & 3^{\prime} \text {-untranslated region } \\ \text { GWAS } & \text { genome-wide association study } \\ \text { LPS } & \text { lipopolysaccharide } \\ \text { FetA } & \text { fetuin-A } \\ \text { MmLDL } & \text { minimally modified low density lipoprotein } \\ \text { PCR-RFLP } & \text { polymerase chain restriction-restriction fragment length polymorphism } \\ \text { OR } & \text { odds ratio } \\ \text { CI } & \text { confidence interval } \\ \text { HWE } & \text { Hardy-Weinberg equilibrium } \\ \text { Chr } & \text { chromosome }\end{array}$

\section{References}

1. Gaziano, T.A.; Bitton, A.; Anand, S.; Abrahams-Gessel, S.; Murphy, A. Growing epidemic of coronary heart disease in low- and middle-income countries. Curr. Probl. Cardiol. 2010, 35, 72-115. [CrossRef] [PubMed]

2. Ma, W.; Xu, J.; Wang, Q.; Xin, Y.; Zhang, L.; Zheng, X.; Wang, H.; Sun, K.; Hui, R.; Huang, X.H. Interaction of functional NPC1 gene polymorphism with smoking on coronary heart disease. BMC Med. Genet. 2010, 11. [CrossRef] [PubMed]

3. Xu, Y.; Wang, W.; Zhang, L.; Qi, L.P.; Li, L.Y.; Chen, L.F.; Fanga, Q.; Dangb, A.-M.; Yana, X.-W. A polymorphism in the ABCG1 promoter is functionally associated with coronary artery disease in a Chinese Han population. Atherosclerosis 2011, 219, 648-654. [CrossRef] [PubMed]

4. Larifla, L.; Maimaitiming, S.; Velayoudom-Cephise, F.L.; Ferdinand, S.; Blanchet-Deverly, A.; Benabdallah, S.; Donnet, J.-P.; Atallah, A.; Roussel, R.; Foucan, L. Association of 2238T > C polymorphism of the atrial natriuretic peptide gene with coronary artery disease in Afro-Caribbeans with type 2 diabetes. Am. J. Hypertens. 2012, 25, 524-527. [CrossRef] [PubMed]

5. Muiya, N.P.; Wakil, S.; Al-Najai, M.; Tahir, A.I.; Baz, B.; Andres, E.; Al-Boudaria, O.; Al-Tassana, N.; Al-Shahidb, M.; Meyera, B.F.; et al. A study of the role of GATA2 gene polymorphism in coronary artery disease risk traits. Gene 2014, 544, 152-158. [CrossRef] [PubMed]

6. Shao, L.; Zhang, P.; Zhang, Y.; Lu, Q.; Ma, A. TLR3 and TLR4 as potential clinically biomarkers of cardiovascular risk in coronary artery disease (CAD) patients. Heart Vessel. 2014, 29, 690-698. [CrossRef] [PubMed]

7. Takeishi, Y.; Kubota, I. Role of Toll like receptor mediated signaling pathway in ischemic heart. Front. Biosci. 2009, 14, 2553-2558. [CrossRef]

8. Michelsen, K.S.; Wong, M.H.; Shah, P.K.; Zhang, W.; Yano, J.; Doherty, T.M.; Akira, S.; Rajavashisth, T.B.; Arditi, M. Lack of Toll like receptor 4 or myeloid differentiation factor 88 reduces atherosclerosis and alters plaque phenotype in mice deficient in apolipoprotein E. Proc. Natl. Acad. Sci. USA 2004, 101, 10679-10684. [CrossRef] [PubMed]

9. Kolek, M.J.; Carlquist, J.F.; Muhlestein, J.B.; Whiting, B.M.; Horne, B.D.; Bair, T.L.; Anderson, J.L. Toll like receptor 4 gene Asp299Gly polymorphism is associated with reductions in vascular inflammation, angiographic coronary artery disease, and clinical diabetes. Am. Heart J. 2004, 148, 1034-1040. [CrossRef] [PubMed]

10. Chen, Z.; Nakajima, T.; Inoue, Y.; Kudo, T.; Jibiki, M.; Iwai, T.; Kimura, A. A single nucleotide polymorphism in the $3^{\prime}$-untranslated region of MyD88 gene is associated with Buerger disease but not with Takayasu arteritis in Japanese. J. Hum. Genet. 2011, 56, 545-547. [CrossRef] [PubMed] 
11. Björkegren, J.L.; Kovacic, J.C.; Dudley, J.T.; Schadt, E.E. Genome-wide significant loci: How important are they? Systems genetics to understand heritability of coronary artery disease and other common complex disorders. J. Am. Coll. Cardiol. 2015, 65, 830-845. [CrossRef] [PubMed]

12. Zhang, K.; Zhang, L.; Zhou, B.; Wang, Y.; Song, Y.; Rao, L.; Zhang, L. Lack of association between TLR4 Asp299Gly polymorphism and atherosclerosis: Evidence from meta-analysis. Thromb. Res. 2012, 130, e203-e208. [CrossRef] [PubMed]

13. Tsai, C.T.; Hwang, J.J.; Ritchie, M.D.; Moore, J.H.; Chiang, F.T.; Lai, L.P.; Hsua, K.-L.; Tsenga, C.-D.; Lina, J.-L.; Tsenga, Y.-Z. Renin-angiotensin system gene polymorphisms and coronary artery disease in a large angiographic cohort: Detection of high order gene-gene interaction. Atherosclerosis 2007, 195, 172-180. [CrossRef] [PubMed]

14. Peng, D.Q.; Zhao, S.P.; Nie, S.; Li, J. Gene-gene interaction of PPARgamma and ApoE affects coronary heart disease risk. Int. J. Cardiol. 2003, 92, 257-263. [CrossRef]

15. Triantafilou, M.; Gamper, F.G.; Lepper, P.M.; Mouratis, M.A.; Schumann, C.; Harokopakis, E.; Schifferle, R.E.; Hajishengallis, G.; Triantafilou, K. Lipopolysaccharides from atherosclerosis-associated bacteria antagonize TLR4, induce formation of TLR2/1/CD36 complexes in lipid rafts and trigger TLR2-induced inflammatory responses in human vascular endothelial cells. Cell. Microbiol. 2007, 9, 2030-2039. [CrossRef] [PubMed]

16. Pal, D.; Dasgupta, S.; Kundu, R.; Maitra, S.; Das, G.; Mukhopadhyay, S.; Sukanta Ray, S.; Majumdar, S.S.; Bhattacharya, S. Fetuin-A acts as an endogenous ligand of TLR4 to promote lipid-induced insulin resistance. Nat. Med. 2012, 18, 1279-1285. [CrossRef] [PubMed]

17. Miller, Y.I.; Choi, S.H.; Wiesner, P.; Bae, Y.S. The SYK side of TLR4: Signalling mechanisms in response to LPS and minimally oxidized LDL. Br. J. Pharmacol. 2012, 167, 990-999. [CrossRef] [PubMed]

18. He, C.; Tu, H.; Sun, L.; Xu, Q.; Li, P.; Gong, Y.; Dong, N.; Yuan, Y. Helicobacter pylori-related host gene polymorphisms associated with susceptibility of gastric carcinogenesis: A two-stage case-control study in Chinese. Carcinogenesis 2013, 34, 1450-1457. [CrossRef] [PubMed]

19. International HapMap Project. HapMap Database (Release 27, Phase I + II + III). Available online: http:/ / www.HapMap.org (accessed on 12 June 2012).

20. Broad Institute. Haploview Software. Available online: http://www.broadinstitute.org/mpg/haploview (accessed on 15 June 2012).

21. National Institutes of Health. FuncPred Software. Available online: http://snpinfo.niehs.nih.gov/snpinfo/ snpfunc.htm (accessed on 16 June 2012).

22. Duan, Z.; He, C.; Gong, Y.; Li, P.; Xu, Q.; Sun, L.P.; Wang, Z.; Xing, C.; Yuan, Y. Promoter polymorphisms in DNA repair gene ERCC5 and susceptibility to gastric cancer in Chinese. Gene 2012, 511, 274-279. [CrossRef] [PubMed]

23. He, C.; Tu, H.; Sun, L.; Xu, Q.; Gong, Y.; Jing, J.; Dong, N.; Yuan, Y. SNP interactions of Helicobacter pylori-related host genes PGC, PTPN11, IL1B, and TLR4 in susceptibility to gastric carcinogenesis. Oncotarget 2015, 6, 19017-19026. [CrossRef] [PubMed]

24. Li, T.; Ren, Z.; Deng, Y.; Wang, Y.; Zhou, H. Lack of association between RAD50-IL13 polymorphisms and pediatric asthma susceptibility in Northeastern Han Chinese. J. Asthma 2015, 52. [CrossRef]

25. Wang, J.Y.; Shyur, S.D.; Lam, F.W.; Wu, L.S. Polymorphisms of EHF-ELF5 genomic region and its association with pediatric asthma in the Taiwanese population. J. Microbiol. Immunol. Infect. 2014, 14. [CrossRef] [PubMed]

26. Xu, Q.; Liu, J.W.; He, C.Y.; Sun, L.P.; Gong, Y.H.; Jing, J.J.; Xing, C.-Z.; Yuan, Y. The interaction effects of pri-let-7a-1 rs10739971 with PGC and ERCC6 gene polymorphisms in gastric cancer and atrophic gastritis. PLoS ONE 2014, 9, e89203. [CrossRef] [PubMed]

27. He, J.; Xiao, Z.; Chen, X.; Chen, M.; Fang, L.; Yang, M.; Lv, Q.; Li, Y.; Li, G.; Hu, J.; et al. The expression of functional Toll like receptor 4 is associated with proliferation and maintenance of stem cell phenotype in endothelial progenitor cells (EPCs). J. Cell. Biochem. 2010, 111, 179-186. [CrossRef] [PubMed]

28. Owens, A.P., III; Rateri, D.L.; Howatt, D.A.; Moore, K.J.; Tobias, P.S.; Curtiss, L.K.; Lu, H.; Cassis, L.A.; Daugherty, A. MyD88 deficiency attenuates angiotensin II-induced abdominal aortic aneurysm formation independent of signaling through Toll like receptors 2 and 4. Arterioscler. Thromb. Vasc. Biol. 2011, 31, 2813-2819. [PubMed] 
29. Yu, M.; Zhou, H.; Zhao, J.; Xiao, N.; Roychowdhury, S.; Schmitt, D.; Hu, B.Q.; Ransohoff, R.M.; Harding, C.V.; Hise, A.G. MyD88-dependent interplay between myeloid and endothelial cells in the initiation and progression of obesity-associated inflammatory diseases. J. Exp. Med. 2014, 211, 887-907. [CrossRef] [PubMed]

30. Miedema, K.G.; te Poele, E.M.; Tissing, W.J.; Postma, D.S.; Koppelman, G.H.; de Pagter, A.P.; Kamps, W.A.; Alizadeh, B.Z.; Boezen, H.M.; de Bont, E.S.J.M. Association of polymorphisms in the TLR4 gene with the risk of developing neutropenia in children with leukemia. Leukemia 2011, 25, 995-1000. [CrossRef] [PubMed]

31. Yamamoto, T.; Tsutsumi, N.; Tochio, H.; Ohnishi, H.; Kubota, K.; Kato, Z.; Shirakawa, M.; Kondo, N. Functional assessment of the mutational effects of human IRAK4 and MyD88 genes. Mol. Immunol. 2014, 58, 66-76. [CrossRef] [PubMed]

32. Xiao, W.; Liu, Z.; Lin, J.; Xiong, C.; Li, J.; Wu, K.; Ma, Y.; Gong, Y.; Liu, Z. Association of TLR4 and TLR5 gene polymorphisms with Graves' disease in Chinese Cantonese population. Hum. Immunol. 2014, 75, 609-613. [CrossRef] [PubMed]

33. Wang, H.; Wei, Y.; Zeng, Y.; Qin, Y.; Xiong, B.; Qin, G.; Li, J.; Hu, D.; Qiu, X.; Sooranna, S.R. The association of polymorphisms of TLR4 and CD14 genes with susceptibility to sepsis in a Chinese population. BMC Med. Genet. 2014, 15. [CrossRef] [PubMed]

34. Sato, K.; Yoshimura, A.; Kaneko, T.; Ukai, T.; Ozaki, Y.; Nakamura, H.; Li, X.Y.; Matsumura, H.; Hara, Y.; Ogata, Y. A single nucleotide polymorphism in $3^{\prime}$-untranslated region contributes to the regulation of Toll like receptor 4 translation. J. Biol. Chem. 2012, 287, 25163-25172. [CrossRef] [PubMed]

35. Milne, R.L.; Fagerholm, R.; Nevanlinna, H.; Benitez, J. The importance of replication in gene-gene interaction studies: Multifactor dimensionality reduction applied to a two-stage breast cancer case-control study. Carcinogenesis 2008, 29, 1215-1218. [CrossRef] [PubMed]

36. Ege, M.J.; Strachan, D.P.; Cookson, W.O.; Moffatt, M.F.; Gut, I.; Lathrop, M.; Kabesch, M.; Genuneit, J.; Büchele, G.; Sozanska, B.; et al. Gene-environment interaction for childhood asthma and exposure to farming in Central Europe. J. Allergy Clin. Immunol. 2011, 127, 138-144. [CrossRef] [PubMed]

37. Wider, C.; Vilarino-Guell, C.; Heckman, M.G.; Jasinska-Myga, B.; Ortolaza-Soto, A.I.; Diehl, N.N.; Crook, J.E.; Cobb, S.A.; Bacon, J.A.; Aasly, J.O.; et al. SNCA, MAPT, and GSK3B in Parkinson disease: A gene-gene interaction study. Eur. J. Neurol. 2011, 18, 876-881. [CrossRef] [PubMed]

38. Jin, M.S.; Lee, J.O. Structures of the toll like receptor family and its ligand complexes. Immunity 2008, 29, 182-191. [CrossRef] [PubMed]

39. McFadden, J.P.; Basketter, D.A.; Dearman, R.J.; Kimber, I.R. Extra domain A-positive fibronectin-positive feedback loops and their association with cutaneous inflammatory disease. Clin. Dermatol. 2011, 29, 257-265. [CrossRef] [PubMed]

40. Kaklikkaya, I.; Kaklikkaya, N.; Buruk, K.; Pulathan, Z.; Koramaz, I.; Aydin, F.; Tosun, I.; Kilic, A.O.; Özcan, F. Investigation of Chlamydia pneumoniae DNA, chlamydial lipopolisaccharide antigens, and Helicobacter pylori DNA in atherosclerotic plaques of patients with aortoiliac occlusive disease. Cardiovasc. Pathol. 2006, 15, 105-109. [CrossRef] [PubMed]

41. Dore, M.P.; Sepulveda, A.R.; Bacciu, P.P.; Blasi, F.; Simula, L.; Marras, L.; Piccolo, D.; Cherchi, G.B.; Graham, D.Y.; Realdi, G. Detection of Chlamydiae pneumoniae but not Helicobacter pylori DNA in atherosclerosis plaques. Dig. Dis. Sci. 2003, 48, 945-951. [CrossRef] [PubMed]

42. Hishiki, S.; Shiwa, T.; Yokoyama, T.; Nomura, M.; Goto, T. Change of cardiovascular risk factors after eradication therapy of Helicobacter pylori. Nihon Shokakibyo Gakkai Zasshi 2001, 98, 814-821. [PubMed]

43. Fedele, F.; Mancone, M.; Chilian, W.M.; Severino, P.; Canali, E.; Logan, S.; de Marchis, M.L.; Volterrani, M.; Palmirotta, R.; Guadagni, F. Role of genetic polymorphisms of ion channels in the pathophysiology of coronary microvascular dysfunctionand ischemic heart disease. Basic Res. Cardiol. 2013, 108, 387. [CrossRef] [PubMed]

(C) 2016 by the authors; licensee MDPI, Basel, Switzerland. This article is an open access article distributed under the terms and conditions of the Creative Commons by Attribution (CC-BY) license (http:/ / creativecommons.org/licenses/by/4.0/). 